\title{
ERRATUM
}

\section{Erratum: PARS2 and NARS2 mutations in infantile- onset neurodegenerative disorder}

Takeshi Mizuguchi, Mitsuko Nakashima, Mitsuhiro Kato, Keitaro Yamada, Tohru Okanishi, Nina Ekhilevitch, Hanna Mandel, Ayelet Eran, Miyuki Toyono, Yukio Sawaishi, Hirotaka Motoi, Masaaki Shiina, Kazuhiro Ogata, Satoko Miyatake, Noriko Miyake, Hirotomo Saitsu and Naomichi Matsumoto

Journal of Human Genetics (2017) 62, 587; doi:10.1038/jhg.2017.13; published online 16 February 2017

Correction to: Journal of Human Genetics (2017) 62, 525-529; doi:10.1038/jhg.2016.163; published online 12 January 2017

In the version of this article initially published online, Supplementary Figure S2 incorrectly appears as Supplementary Figure S1. This error has now been rectified and the correct Supplementary Figure S1 appears in this erratum.

The publisher would like to apologize for this error and any inconvenience it may have caused.
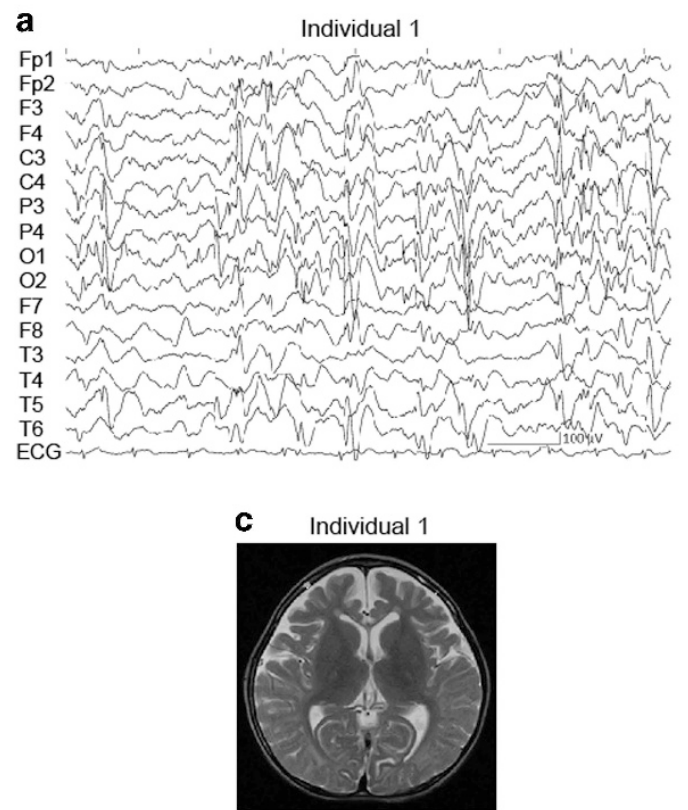
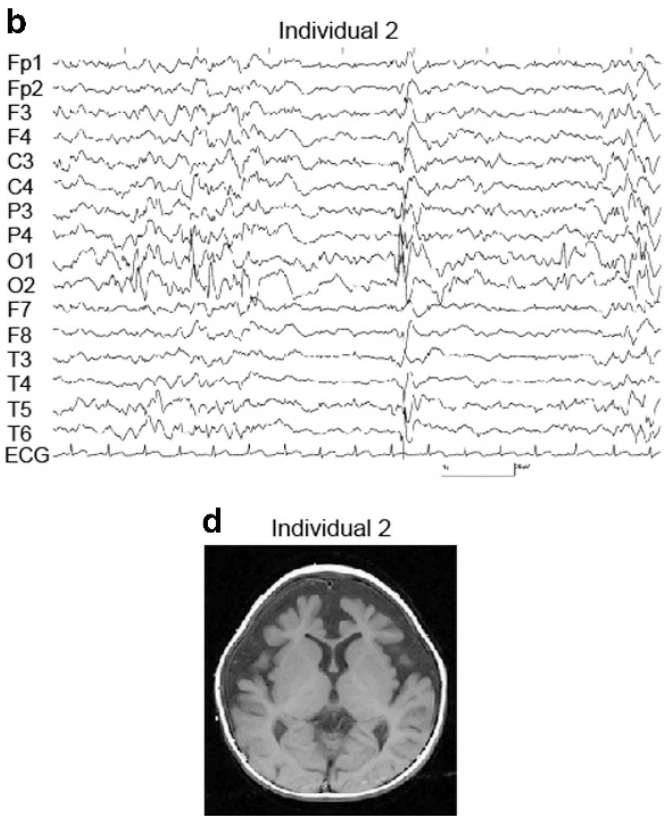

Supplementary Figure S1. EEG and brain MRI of patients with PARS2 mutations. (a) EEG of Individual 1 at 3 years and 5 months of age. Multifocal, hemispherical spikes, polyspikes, sharp waves, and sharp-and-slow waves are seen with a high-amplitude slow wave background, which is compatible to hypsarrhythmia. (b) EEG of Individual 2 at 4 months of age shows sharp waves or sharp-and-slow waves at $\mathrm{O} 1$ or $\mathrm{O} 2$ region and diffuse propagation of irregular polyspike-and-slow wave. (c) Brain MRI of Individual 1 at 7 years of age. Axial view of T2-weighted image shows abnormal high intensity on the subcortical and deep white matter suggesting delayed myelination except for the outer and internal capsule and optic radiation. Diffusely wide subarachnoidal space, decreased volume of the frontal lobes, thin corpus callosum, and mildly dilated posterior horns of the lateral ventricle are also seen. (d) Brain MRI of Individual 2 at 16 months of age. Axial view of T1-weighted image shows blurred boundary between the cortex and white matter suggesting delayed myelination. Wide subarachnoidal space and decreased volume of the frontal lobes with thin genu of the corpus callosum are also seen. 